\title{
Comparative study of indocyanine green combined with blue dye with methylene blue only and carbon nanoparticles only for sentinel lymph node biopsy in breast cancer
}

\author{
Xingsong Qin, Muwen Yang, Xinyu Zheng \\ Department of Breast Surgery, First Affiliated Hospital, China Medical University, Shenyang, China
}

\begin{abstract}
Purpose: The combination of indocyanine green and methylene blue (ICG + MB) was reported to be an efficient tracer method in sentinel lymph node biopsy (SLNB). However, whether this method is superior to MB only or carbon nanoparticles (CN) is controversial. This study was to evaluate the efficacy of the three methods in SLNB for breast cancer, and to analyze its influencing factors.

Methods: One hundred eighty patients with early breast cancer were recruited and randomly divided into 3 groups. Each group comprising of 60 patients with SLNB using ICG + MB, MB, and CN, respectively. Then the 3 groups were compared in detection rate, mean number of SLNs, and the detection rates and number of metastatic sentinel lymph nodes (SLNs).

Results: The detection rate of SLNs was $100 \%$ (60 of 60 ) in ICG + MB group, $96.7 \%$ (58 of 60 ), and $98.3 \%$ (59 of 60 ) in MB and $\mathrm{CN}$ group, respectively, with no significant difference $(\mathrm{P}=0.362$ ). Totally, $204 \mathrm{SLNs}$ (mean \pm standard deviation [SD] [range], $3.4 \pm 1.4$ [2-8]) were detected in ICG + MB group, 102 (1.7 \pm 0.7 [0-3]) and 145 (2.4 \pm 0.7 [0-6]) in MB and CN group, indicating significant difference $(P<0.001)$. The detection rate of metastatic SLN was $23.3 \%$ (14 of 60$)$ in ICG + MB group, which was higher than $18.3 \%$ (11 of 60 ) and $20 \%$ (11 of 60) in MB and CN group, respectively, but showed no statistical significance $(P=0.788)$.

Conclusion: ICG + MB method was superior to MB only and CN only methods in the mean number of SLNs, thus predicting axillary lymph node metastasis more accurately. Therefore, in areas where the standard method is not available, ICG + MB may be more suitable as an alternative tracer for SLNB.

[Ann Surg Treat Res 2019;97(1):1-6]
\end{abstract}

Key Words: Carbon nanoparticles, Indocyanine green, Methylene blue, Sentinel lymph node biopsy

\section{INTRODUCTION}

The knowledge of regional lymph node status is essential for staging the disease and predicting its prognosis [1]. Sentinel lymph node biopsy (SLNB) has been generally accepted and applied in surgical treatment of various carcinomas for many years $[2,3]$. SLNB for patients with early breast cancer recommended is the primary treatment for patients with no clinical evidence of axillary lymph node metastasis by American Society of Clinical Oncology [4]. The internationally recommended standard tracer method for SLNB is the combination of radioisotope and blue dye, which identifies sentinel lymph nodes (SLNs) at a rate of more than $95 \%$, and a false-negative rate of below $10 \%[5,6]$. However, despite
Received November 13, 2018, Revised April 1, 2019,

Accepted April 12, 2019

Corresponding Author: Xinyu Zheng

Department of Breast Surgery, First Affiliated Hospital, China Medical University, 155 North Nanjing Street, Shenyang, Liaoning 110001, China

Tel: +86-24-83282741, Fax: +86-24-83282773

E-mail:xyzheng@cmu.edu.cn

ORCID code: https://orcid.org/0000-0001-8425-3379
Copyright (C) 2019, the Korean Surgical Society

(c) Annals of Surgical Treatment and Research is an Open Access Journal. All articles are distributed under the terms of the Creative Commons Attribution NonCommercial License (http://creativecommons.org/licenses/by-nc/4.0/) which permits unrestricted non-commercial use, distribution, and reproduction in any medium, provided the original work is properly cited. 
its effectiveness, the use of radioactive tracers brings many challenges, including the risk of exposure to radiation, staff training and legislative requirements [7]. Consequently, this gold standard method is not feasible in many medical institutions, especially in developing countries like China. Interestingly, widespread use of blue dye and carbon nanoparticle has been observed in these areas, though their performances have been reported to be not consistent and different [8-14].

The combination of indocyanine green (ICG) and blue dye was recently introduced and reported to be a highly sensitive method for SLN detection. Two studies published in 2017 suggested the detection rate of the new method was close to $100 \%$, and the number of SLNs detected was near $4[15,16]$. Despite that, one trial performed by Samorani et al. [17] suggested that $100 \%$ detection of SLN was observed using only ICG without combining it with any other tracers. So, it still remains controversial whether the combination of ICG and blue dye is superior to dye-only method.

Therefore, based on these domestic widespread resources, our study tried to investigate whether the combination of ICG and methylene blue (MB) could perform better than $\mathrm{MB}$ or carbon nanoparticles $(\mathrm{CN})$, in terms of the detection rate of SLNs, number of SLNs, and the detection rate of metastatic SLNs. This information will help clinicians find an alternative non-radioactive method for management of breast cancer in the areas where the standard method is not available.

\section{METHODS}

\section{Patients}

In this prospective study, a total of 180 patients with early breast cancer were enrolled between October 2016 and December 2017 at our hospital. The patient inclusion criteria consisted of pathological diagnosis of breast cancer, maximum tumor diameter of $5 \mathrm{~cm}$, no identification of clinically metastatic axillary lymph nodes through ultrasound, chest computed tomography or breast magnetic resonance imaging. Also, the exclusion criteria included palpable axillary lymph nodes, previous axillary surgery, neoadjuvant chemotherapy or endocrine therapy, inflammatory breast cancer, multicentric tumor, and pregnancy. After recruitment, patients were randomized into 3 groups. Each group comprising of 60 patients had SLNB using ICG + MB, MB, and CN, respectively.

This study strictly followed the Helsinki Declaration guidelines, and was approved by the Institutional Review Board of First Affiliated Hospital of China Medical University (approval number: 2016QL011). Every patient provided written informed consent, before surgery.

\section{Surgical procedure}

SLN identification using ICG + MB: Five minutes before surgery, $1 \mathrm{~mL}$ of $1 \%$ methylene blue dye (Jichuan Pharmaceutical, Taizhou, China) was injected subdermally into the periareolar region, and 4 minutes later, $1 \mathrm{~mL}$ of indocyanine green solution (Yichuang Pharmaceutical LLC, Dandong, China) was injected into the same region intradermally. Following gentle breast
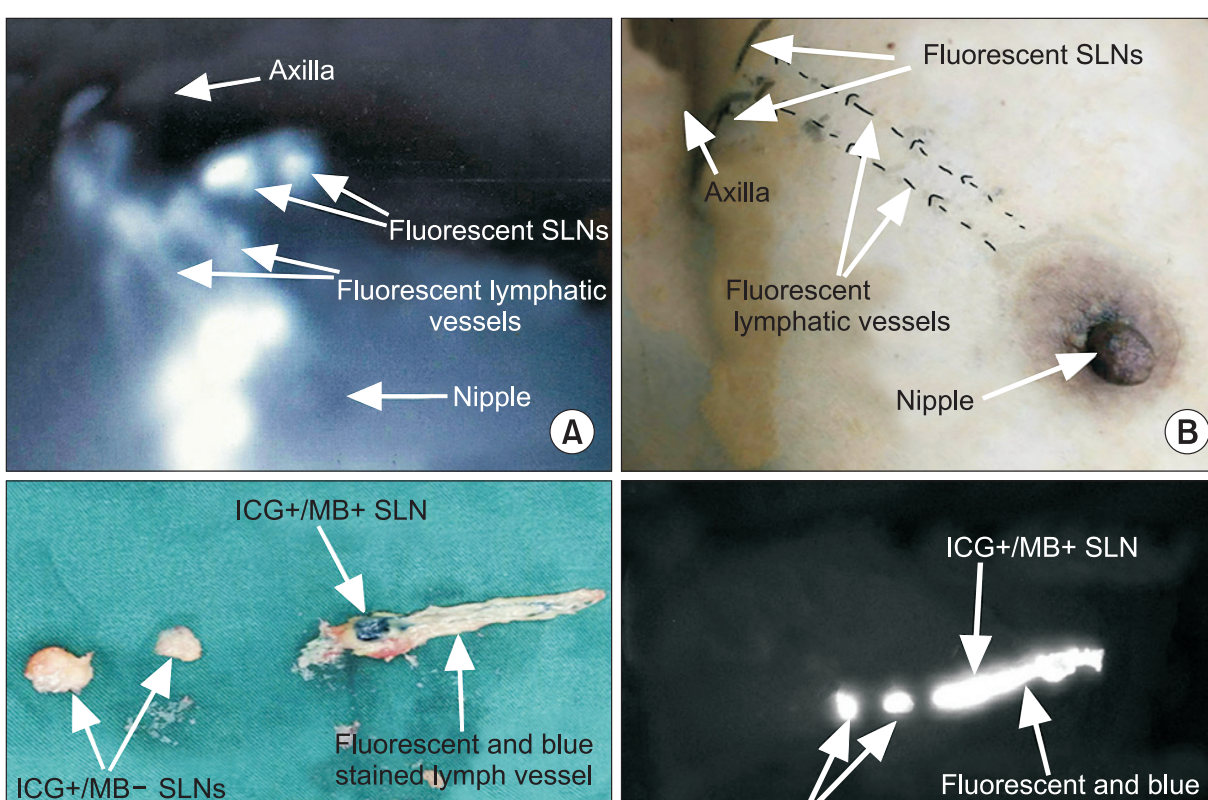

(C)
Fig. 1. Sentinel lymph node biopsy using ICG + MB method. (A) Two streams of fluorescent dermal lymphatic vessels and 2 fluorescent nodes were clearly observed by photodynamic eye camera. (B) The fluorescent lymphatic vessels and SLN flow were marked on the skin and an incision was made upon SLN region. (C) Three nodes and 1 lymphatic vessel link were removed. Only 1 was blue-stained. (D) All SLNs and the lymphatic vessels were fluorescent under the photodynamic eye camera. The result: 1 was ICG+/MB+ and 2 SLNs were ICG+/MB-. ICG, indocyanine green; $M B$, methylene blue; SLN, sentinel lymph node. 

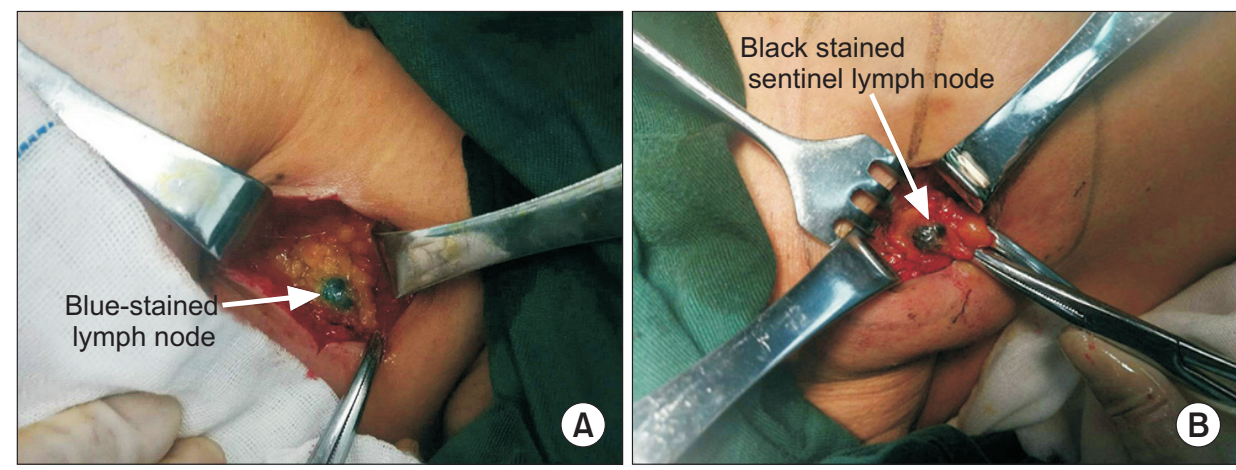

Fig. 2. Sentinel lymph node biopsy using $\mathrm{MB}$ and $\mathrm{CN}$, respectively. (A) One blue-stained lymph node was detected in $\mathrm{MB}$ group. (B) One black-stained lymph node was detected in $\mathrm{CN}$ group. $\mathrm{MB}$, methylene blue; $\mathrm{CN}$, carbon nanoparticles.

massage, the lymphatic flow pathway was observed using photodynamic eye camera (MingDe Biomedical Technology Corp., Hebei, China). Later, a skin incision was made at a point where fluorescent nodes appeared to detect lymph node (Fig. 1A, B). Subsequently, all removed SLNs were separately examined, and classified into 3 categories: (1) simultaneously fluorescent and blue-stained (ICG+/MB+), (2) only fluorescent (ICG+/MB-), and (3) only blue-stained (ICG-/MB+) (Fig. 1C, D).

\section{SLNs identification using $M B$}

Five minutes before surgery, $1 \mathrm{~mL}$ of $\mathrm{MB}$ dye was injected subdermally into the periareolar region of 4 quadrants of the breast. Following gentle massage, a small transverse incision was made one centimeter below the axillary line to detect stained lymph-vessels. Then, the axilla was inspected for bluestained nodes (Fig. 2A).

\section{SLNs identification using CN}

Ten to fifteen minutes before surgery, $1 \mathrm{~mL}$ of $\mathrm{CN}$ suspension (Chongqing LUMMY Pharmaceutical, Chongqing, China) was intradermally injected into the periareolar region. The whole breast was massaged for about 5 minutes to facilitate the absorption of $\mathrm{CN}$ into the lymph vessels. Later, a transverse incision was made in the same position as was done in the MB group. After raising the skin flap, black-stained SLNs were detected (Fig. 2B).

All these SLNs were examined by intraoperative histopathology examination of sliced 2-mm sections of frozen tissues to detect SLN metastasis. In patients displaying malignant cells in the SLN of frozen sections, axillary lymph node dissection (ALND) was performed as a standard axillary treatment.

\section{Statistical method}

Variables between 3 methods were compared using chisquare test and independent sample t-test. All statistical analyses were performed using IBM SPSS Statistics ver. 23.0 (IBM Co., Armonk, NY, USA) software, and P-value of $<0.05$ represented statistical significance.
Table 1. Patients and tumor characteristics

\begin{tabular}{|c|c|c|c|c|}
\hline Characteristic & $\begin{array}{c}\mathrm{ICG}+ \\
\mathrm{MB} \\
(\mathrm{n}=60)\end{array}$ & $\begin{array}{c}M B \\
(n=60)\end{array}$ & $\begin{array}{c}C N \\
(n=60)\end{array}$ & P-value \\
\hline Age $(y r)$ & & & & 0.547 \\
\hline$\leq 50$ & 35 & 29 & 32 & \\
\hline$>50$ & 25 & 31 & 28 & \\
\hline Body mass index $\left(\mathrm{kg} / \mathrm{m}^{2}\right)$ & & & & 0.435 \\
\hline$\leq 18.4$ & 2 & 1 & 1 & \\
\hline $18.5-23.9$ & 36 & 26 & 28 & \\
\hline $24.0-27.9$ & 18 & 23 & 24 & \\
\hline$\geq 28$ & 4 & 10 & 7 & \\
\hline Histologic type & & & & 0.905 \\
\hline Invasive ductal & 46 & 49 & 51 & \\
\hline Invasive lobular & 1 & 1 & 1 & \\
\hline DCIS & 10 & 7 & 7 & \\
\hline Other types & 3 & 3 & 1 & \\
\hline Tumor size $(\mathrm{cm})$ & & & & 0.537 \\
\hline$<2.0$ & 48 & 43 & 44 & \\
\hline $2-5$ & 12 & 17 & 16 & \\
\hline Menopause & & & & 0.344 \\
\hline Pre & 34 & 26 & 30 & \\
\hline Post & 26 & 34 & 30 & \\
\hline Tumor stage & & & & 0.425 \\
\hline T0 & 12 & 8 & 7 & \\
\hline $\mathrm{T} 1$ & 27 & 30 & 30 & \\
\hline $\mathrm{T} 2$ & 21 & 20 & 23 & \\
\hline T3 & 0 & 2 & 0 & \\
\hline Tumor localization & & & & 0.868 \\
\hline Upper outer & 26 & 31 & 28 & \\
\hline Lower outer & 10 & 9 & 12 & \\
\hline Upper medial & 20 & 17 & 15 & \\
\hline Lower medial & 3 & 1 & 4 & \\
\hline Central & 1 & 2 & 1 & \\
\hline Prior biopsy & & & & 0.672 \\
\hline Excisional biopsy & 45 & 48 & 44 & \\
\hline Core needle biopsy & 15 & 12 & 16 & \\
\hline Estrogen receptor status & & & & 0.901 \\
\hline Positive & 49 & 47 & 48 & \\
\hline Negative & 11 & 13 & 12 & \\
\hline
\end{tabular}

ICG, indocyanine green; $\mathrm{MB}$, methylene blue; $\mathrm{CN}$, carbon nanoparticles; DCIS, ductal carcinoma in situ. 


\section{RESULTS}

\section{Patient and tumor characteristics}

The detailed information about the patient characteristics and tumors has been shown in Table 1. There was no difference among mean age, body mass index, histological classification, menopause status, and $\mathrm{T}$ stage or tumor localization in patients from all 3 groups.

\section{SLN detection by different methods}

The detection rates of the three groups are shown in Table 2. The SLN detection rates for the ICG $+\mathrm{MB}, \mathrm{MB}$, and $\mathrm{CN}$ were $100 \%, 96.7 \%$, and $98.3 \%$, respectively ( $P>0.05$ ). All patients in ICG + MB group were observed when the fluorescent lymphvessel flow in the skin was visible before skin resection, and were detected with fluorescent SLNs after transcutaneous fluorescent lymphography. Fifty-eight of the 60 patients in the MB group showed blue stained nodes, and 59 patients in the $\mathrm{CN}$ group had their SLNs successfully stained black after skin and subdermal fat incision.

A total of 204 SLNs were removed from patients in ICG + MB group (101 with ICG+/MB+; 98 with ICG+/MB-; 5 with ICG-/ $\mathrm{MB}+)$. The total number of SLNs was 102 and 145 in MB and $\mathrm{CN}$ group, respectively. In addition, the mean number of SLNs was $3.4 \pm 1.4$ (range, 2-8), $1.7 \pm 0.7$ (range, $0-3$ ), and $2.4 \pm 0.7$ (range, $0-6)$ in ICG $+\mathrm{MB}, \mathrm{MB}$, and $\mathrm{CN}$ groups, respectively $(\mathrm{P}<$ 0.001) (Table 2).

\section{Metastasis rate of SLNs in different groups}

Among the total 60 patients analyzed in the ICG + MB group, 14 tumor-positive patients were detected with 18 metastatic SLNs in the frozen sections (11 with ICG+/MB+; 6 with ICG+/ $\mathrm{MB}-; 1$ with ICG-/MB+), and there were 11 positive patients with 13 metastatic SLNs and 12 positive patients with 15 metastatic SLNs in the MB and CN group, respectively. The detection rate of metastatic SLNs in ICG + MB group was 23.3\%, higher than that of $\mathrm{MB}(18.3 \%)$ and $\mathrm{CN}$ group (20.0\%), respectively, but not statistically significant $(\mathrm{P}>0.05)$ (Table 2).

\section{DISCUSSION}

Typically, the ideal SLN tracer method is supposed to not only possess advantages such as high detection rate, sensitivity, and acceptable false negative rate, but also should meet the requirements of simple operation, safety, and low cost. Despite the combination of radioisotope and blue dye being recommended as the gold standard method for SLNB [6], there are certain limitations for its generalization [7]. Therefore, in the areas where radioactive isotopes are not available, other alternative methods are urgently needed. Currently, there are more than 5 tracer alternative resources that have been used for SLNB, including blue dye, CN, indocyanine green, contrastenhanced ultrasound using microbubbles, superparamagnetic iron oxide nanoparticles, and other combination of various tracers. In China, $\mathrm{CN}$ and methylene blue are the most widely used and studied.

Our present study was designed to evaluate the performance of the 3 different methods for SLNB. The results demonstrated that despite similar SLN detection rates in the 3 groups, the mean number of traced SLNs removed in ICG + MB group was approximately 2 times that in MB group and one more than that in $\mathrm{CN}$ group. Similar results have also been observed in other previously published studies [12,15]. Actually, the numbers of removed SLNs were informative in predicting metastasis in axillary lymph node. According to previous studies [18-20], the increase in the number of removed SLNs would result in enhanced accuracy and decline in false negative rate. Particularly, when SLNs detection number reaches 4 , the accuracy rate can be stabilized at $95 \%$ and the false negative rate can be controlled below $5 \%$. The relationship between the number of removed SLNs and false negative rate $\&$ accuracy has also been shown in Table 3 .

To identify reasons that caused enhanced detection of traced lymph nodes in ICG + MB group, it appeared that ICG and $\mathrm{MB}$ can complement each other's disadvantages. ICG typically enables visualization of fluorescent flow through lymph-vessels into the nodes, and thus helps doctors to quickly locate lymph nodes [21,22]. But the disadvantage is leakage, caused by cuts in the lymphatic ducts after the excision of the first node, which makes it difficult to detect other fluorescent nodes in the surgical field. To complement this, surgeons can continue to search blue-stained nodes by naked eye. Indeed, 5 SLNs (2.1\%) in ICG + MB group were only stained blue, thereby indicating that some nodes may have escaped if ICG was used alone. This

Table 2. Comparison of sentinel lymph node biopsy results in the 3 groups

\begin{tabular}{lcccc}
\hline \multicolumn{1}{c}{ Characteristic } & ICG + MB $(\mathrm{n}=60)$ & MB $(\mathrm{n}=60)$ & $\mathrm{CN}(\mathrm{n}=60)$ & $\mathrm{P}$-value \\
The detection rate of SLNs & $60(100)$ & $58(96.7)$ & $59(98.3)$ & 0.362 \\
Mean number of SLNs & $3.4 \pm 1.4$ & $1.7 \pm 0.7$ & $2.4 \pm 0.7$ & $<0.001$ \\
The detection rate of metastatic SLNs & $14(23.3)$ & $11(18.3)$ & $12(20.0)$ & 0.788 \\
\hline
\end{tabular}

Values are presented as number (\%) or mean \pm standard deviation.

$\mathrm{SLN}$, sentinel lymph nodes; ICG, indocyanine green; MB, methylene blue; $\mathrm{CN}$, carbon nanoparticles. 
Table 3. Relationship between sentinel lymph nodes (SLNs) removed and false negative rate \& accuracy

\begin{tabular}{|c|c|c|c|c|c|}
\hline \multirow{2}{*}{ Study } & \multirow{2}{*}{ Characteristic } & \multicolumn{4}{|c|}{ SLNs removed $(\mathrm{n})$} \\
\hline & & 1 & 2 & 3 & $\geq 4$ \\
\hline \multirow[t]{2}{*}{ Guo et al. [16] } & False negative rate $(\%)$ & 18.2 & 11.6 & 10.8 & 1.9 \\
\hline & Accuracy (\%) & 91.2 & 93.4 & 94.0 & 98.7 \\
\hline \multirow[t]{2}{*}{ Ban et al. [19] } & False negative rate $(\%)$ & 10.8 & 5.0 & 3.5 & 0.0 \\
\hline & Accuracy $(\%)$ & 96.3 & 98.3 & 98.7 & 100.0 \\
\hline \multirow[t]{2}{*}{ Koo et al. [20] } & False negative rate $(\%)$ & 23.1 & 11.1 & 4.8 & 0.0 \\
\hline & Accuracy (\%) & 90.9 & 96.2 & 97.9 & 100.0 \\
\hline
\end{tabular}

number of SLNs with ICG-/MB+ seems so few that they could be neglected, but it cannot be ignored since we cannot confirm whether there is no metastatic SLN among them. In fact, one of the 18 metastatic SLNs in the ICG + MB group was detected with MB only.

Furthermore, the total number of fluorescent SLNs in ICG + MB group were much more than blue-stained and black-stained SLNs in MB and CN groups, respectively (199 vs. 102 vs. 145). Inside the ICG $+\mathrm{MB}$ group, fluorescent lymph nodes were also observed to be more than blue-stained nodes (199 vs. 106). This difference can be attributed to the fact that ICG has a stronger affinity for the lymphatic system than $\mathrm{MB}$ and $\mathrm{CN}$, and this affinity may be caused by molecular structure and diameter rather than molecular mass, as molecular mass of ICG (774.9) is larger than MB (319.9) and CN (12.1) [10,23]. Besides that, the visual interference caused by intraoperative bleeding will obstruct SLN identification in both MB and CN methods, particularly in obese patients [10].

As for the patients who were not detected with any traced SLNs, there may be multiple reasons for the failure of tracing. Degeneration of lymphoid tissue with aging, disruption of lymphatic drainage due to previous tumor-excision, and importantly, obesity, can be a few of these reasons. In our study, 2 patients in MB group and 1 in CN group were not identified with blue or black stained SLNs. Upon reviewing the characteristics of these patients, 2 had stage IIA invasive ductal carcinoma with tumor size $10-15 \mathrm{~mm}$, and the tumors located on the upper outer side and were excised in previous surgery. So, it is possible that lymphatic vessels were cut and the tracer failed to reach the SLNs. However, the third patient was a 63-year-old woman with upper/outer 15- to 20-mm tumor, and her tumor histology was encapsulated papillary carcinoma, which may have more probability of tumor thrombus blocking the lymphatics.

Even the detection rates of metastatic SLNs among the three groups showed no statistically significant difference; the number of positive patients in ICG + MB group was more than that in $\mathrm{MB}$ and $\mathrm{CN}$ groups. Furthermore, current clinical guidelines for breast cancer stipulate that ALND should be performed only on patients who had sentinel node involved by tumor, while patients without any positive node would no longer undergo additional ALND. So, our study could not confirm the false negative rate or specificity adequately. However, it is why we highlight the number of SLNs which have a direct impact on the accuracy. Studies have confirmed that the number of SLN detection directly affects the false negative rate and accuracy; that is, with the increase of the number of SLN detection, false negative rate will be reduced and accuracy will be enhanced. The false negative rate can be controlled below $11 \%$ and the accuracy rate can be over $94 \%$ when the number of SLNs reaches 3 . When 4 SLNs were detected, the false negative rate was below $2 \%$, and the accuracy rate was above $98 \%$ [18-20].

It could be explained by the fact that positive SLNs are more likely to be detected as the number of nodes increases. According to a study authored by Yi et al. [24], greater than $96 \%$ of positive SLNs were identified in the first 3 lymph nodes removed. We could safely conclude that the number matters.

Our current study also had several shortcomings. First, we did not compare the 3 methods with the standard method. Thus, it is hard to conclude if any of the analyzed methods were equivalent to the gold standard. In addition, we did not have long enough follow-up, and consequently failed to confirm cancer recurrence in axillary lymph nodes. Third, in the absence of ALND for all patients, our study could not confirm false negative rate or specificity adequately.

In summary, although there was no statistical significance on the detection rate of SLN and the detection rate of metastatic SLN, the ICG + MB method was definitely superior to MB and $\mathrm{CN}$ in terms of the mean number of SLNs, which may enhance accuracy and reduce false negative rate in SLNB. Based on our study, it is proposed that the combination of ICG and MB method can be the most likely alternative to the existing gold standard in institutions where radioactive agents are not available.

\section{CONFLICTS OF INTEREST}

No potential conflict of interest relevant to this article was reported. 
1. Goldhirsch A, Wood WC, Gelber RD, Coates AS, Thurlimann B, Senn HJ, et al. Progress and promise: highlights of the international expert consensus on the primary therapy of early breast cancer 2007. Ann Oncol 2007;18:1133-44.

2. Paek SH, Yi KH, Kim SJ, Choi JY, Lee KE, Park YJ, et al. Feasibility of sentinel lymph node dissection using Tc-99m phytate in papillary thyroid carcinoma. Ann Surg Treat Res 2017:93:240-5.

3. Oh SY, Kim DY, Kim YB, Suh KW. Clinical application of sentinel lymph node mapping in colon cancer: in vivo vs. ex vivo techniques. Ann Surg Treat Res 2014;87: 118-22.

4. Lyman GH, Somerfield MR, Giuliano AE. Sentinel lymph node biopsy for patients with early-stage breast cancer: 2016 American Society of Clinical Oncology Clinical Practice Guideline Update Summary. J Oncol Pract 2017;13:196-8.

5. van der Vorst JR, Schaafsma BE, Verbeek FP, Hutteman M, Mieog JS, Lowik CW, et al. Randomized comparison of nearinfrared fluorescence imaging using indocyanine green and $99(\mathrm{~m})$ technetium with or without patent blue for the sentinel lymph node procedure in breast cancer patients. Ann Surg Oncol 2012;19: 4104-11.

6. Lyman GH, Giuliano AE, Somerfield MR, Benson AB 3rd, Bodurka DC, Burstein HJ, et al. American Society of Clinical Oncology guideline recommendations for sentinel lymph node biopsy in early-stage breast cancer. J Clin Oncol 2005;23:770320.

7. Stratmann SL, McCarty TM, Kuhn JA. Radiation safety with breast sentinel node biopsy. Am J Surg 1999;178:454-7.

8. Wang X, Liu J, Hou Y, Wang N, Wang M. Logistic regression analysis for factors affecting the successful rate of nanocarbon in sentinel lymph node biopsy.
Zhong Nan Da Xue Xue Bao Yi Xue Ban 2016;41:411-6.

9. Varghese P, Abdel-Rahman AT, Akberali S, Mostafa A, Gattuso JM, Carpenter R. Methylene blue dye--a safe and effective alternative for sentinel lymph node localization. Breast J 2008;14:61-7.

10. Wu X, Lin Q, Chen G, Lu J, Zeng Y, Chen $X$, et al. Sentinel lymph node detection using carbon nanoparticles in patients with early breast cancer. PLoS One 2015; 10:e0135714.

11. Simmons R, Thevarajah S, Brennan MB, Christos P, Osborne M. Methylene blue dye as an alternative to isosulfan blue dye for sentinel lymph node localization. Ann Surg Oncol 2003;10:242-7.

12. Sugie T, Sawada T, Tagaya N, Kinoshita T, Yamagami K, Suwa H, et al. Comparison of the indocyanine green fluorescence and blue dye methods in detection of sentinel lymph nodes in early-stage breast cancer. Ann Surg Oncol 2013;20:2213-8.

13. Ahmed M, Purushotham AD, Douek M. Novel techniques for sentinel lymph node biopsy in breast cancer: a systematic review. Lancet Oncol 2014;15:e351-62.

14. Ang CH, Tan MY, Teo C, Seah DW, Chen JC, Chan MY, et al. Blue dye is sufficient for sentinel lymph node biopsy in breast cancer. Br J Surg 2014;101:383-9.

15. Ji Y, Luo N, Jiang Y, Li Q, Wei W, Yang H, et al. Clinical utility of the additional use of blue dye for indocyanine green for sentinel node biopsy in breast cancer. J Surg Res 2017;215:88-92.

16. Guo J, Yang H, Wang S, Cao Y, Liu M, Xie F, et al. Comparison of sentinel lymph node biopsy guided by indocyanine green, blue dye, and their combination in breast cancer patients: a prospective cohort study. World J Surg Oncol 2017;15:196.

17. Samorani D, Fogacci T, Dellachiesa L. Use of indocyanine green alone for sentinel node biopsy in breast cancer. Breast Can- cer 2015;22:442-3.

18. Yang B, Zheng G, Zuo WS, Yang L, Wang YS, Zheng MZ, et al. Analysis of clinicopathological factors associated with false-negative rate of sentinel lymph node biopsy in breast cancer patients: experience of a single center. Zhonghua Zhong Liu Za Zhi 2013:35:389-93.

19. Ban EJ, Lee JS, Koo JS, Park S, Kim SI, Park BW. How many sentinel lymph nodes are enough for accurate axillary staging in t1-2 breast cancer? J Breast Cancer 2011;14: 296-300.

20. Koo BY, Jeong SG, Eom TI, Kang HJ, Kim LS. The number of removed lymph nodes for an acceptable false negative rate in sentinel lymph node biopsy for breast cancer. J Breast Cancer 2009;12:100-5.

21. Sugie T, Kassim KA, Takeuchi M, Hashimoto T, Yamagami K, Masai Y, et al. A novel method for sentinel lymph node biopsy by indocyanine green fluorescence technique in breast cancer. Cancers (Basel) 2010;2:713-20.

22. Takeuchi M, Sugie T, Abdelazeem K, Kato H, Shinkura N, Takada M, et al. Lymphatic mapping with fluorescence navigation using indocyanine green and axillary surgery in patients with primary breast cancer. Breast J 2012;18:535-41.

23. Hutteman M, Mieog JS, van der Vorst JR, Liefers GJ, Putter H, Lowik CW, et al. Randomized, double-blind comparison of indocyanine green with or without albumin premixing for near-infrared fluorescence imaging of sentinel lymph nodes in breast cancer patients. Breast Cancer Res Treat 2011;127:163-70.

24. Yi M, Meric-Bernstam F, Ross MI, Akins JS, Hwang RF, Lucci A, et al. How many sentinel lymph nodes are enough during sentinel lymph node dissection for breast cancer? Cancer 2008;113:30-7. 